\title{
REVISIÓN DE GUÍAS PARA AVANZAR HACIA UNA EDUCACIÓN MÁS INCLUSIVA EN LOS CENTROS ESCOLARES
}

\section{Review of Guidelines for moving towards more Inclusive Education in Schools}

\author{
Cecilia Azorín Abellán \\ Universidad de Murcia \\ cmaria.azorin@um.es \\ Mónica del Mar González Botía \\ Universidad de Murcia
}

Recepción: 5 de junio de 2020

Aceptación definitiva: 19 de octubre de 2020

Resumen: En los últimos años, ha habido un incremento exponencial en el número de guías, materiales e informes que se encuentran a disposición de los centros escolares para orientar su camino hacia la inclusión. Partiendo de esta realidad, se ha llevado a cabo un estudio de naturaleza cualitativa cuyo objetivo principal ha consistido en una selección de guías sobre inclusión que resultan de utilidad para iniciar procesos de reflexión-acción en la comunidad educativa. El análisis documental de contenido (de un total de 13 guías) ha permitido identificar los facilitadores/limitadores que posibilitan/ dificultan el avance de la inclusión. Asimismo, se han especificado los recursos/instrumentos que ofrecen las guías para acompañar a los centros en el camino hacia una educación más inclusiva. Los resultados recogen la importancia que tiene la formación del profesorado en la atención a la diversidad, la puesta en marcha de redes de colaboración y la reformulación de barreras en facilitadores. Las conclusiones apuntan a la necesidad de iniciar/sostener procesos de mejora para una mayor inclusión dentro y fuera de las aulas y de dar a conocer este corpus de guías a los profesionales que están en la práctica.

Palabras Clave: educación inclusiva; análisis documental; investigación educativa; mejora escolar. 
АвSTRACT: In recent years, a growing number of guides and reports have become available for schools to guide them on their paths towards inclusion. A qualitative study has been carried out, whose main aim was to select guides on inclusion that are useful for initiating reflection-action processes within educational community. The documentary analysis (of 13 guides in all) led to the identification of the facilitators/barriers that favor/hinder the advance of inclusion. Likewise, the resources/instruments offered by the guides have been specified in order to support schools on their journeys towards a more inclusive education. The results reflect the importance of teacher training in dealing with diversity, the implementation of collaborative networks and the reformulation of barriers into facilitators. The conclusions point to the need to initiate/sustain improvement processes for greater inclusion within and outside the classroom, and to make this corpus of guidance known to practitioners.

KEYwORDs: inclusive education; documentary analysis; educational research; school improvement.

\section{Todo por la inclusión, pero sin la inclusión}

L

A INCLUSIÓN TIENE QUE VER CON LA ELIMINACión de los procesos excluyentes manifiestos en determinadas actitudes y respuestas hacia la diversidad, en ocasiones, motivadas por razón de género, logro, etnia, clase social, religión, orientación sexual y discapacidad, entre otros (Ainscow, 2015). Asimismo, la inclusión es un proceso que aspira a mejorar la respuesta a la diversidad del alumnado (con y sin necesidades educativas especiales) y se compromete también con el alumnado en riesgo de marginación, exclusión o fracaso escolar (Ainscow, 2017). Por consiguiente, como aclara Echeita (2017), la inclusión no trata de responder exclusivamente al alumnado en situación de riesgo o discapacidad, sino que se dirige a todas las personas, con independencia de sus características culturales, sociales, biológicas, afectivas, intelectuales o de cualquier otra índole.

La Agenda 2030 (UNESCO, 2015) ha abanderado el compromiso de proporcionar una educación más eficaz, eficiente, inclusiva y equitativa. Sin embargo, Calderón (2018) advierte que en la mayoría de las escuelas españolas existe una exclusión institucionalizada, lo que descarta la idea de inclusión real en este país. Desde una panorámica de análisis crítico, Azorín y Sandoval (2019) han acuñado el término "despotismo inclusivo" para referirse a la teoría sobre la inclusión que cabalga por un lado y a la realidad de la práctica que lo hace por otro muy diferente, adoptando así el enfoque del todo por la inclusión (teoría), pero sin una inclusión aparentemente tangible en las aulas (práctica).

En este contexto, Ainscow (2020) afirma que los centros educativos han de ser capaces de iniciar y sostener procesos de mejora e innovación para que la inclusión realmente se materialice. Así pues, adquiere gran relevancia el reconocimiento de las barreras que limitan el desarrollo de la inclusión y la identificación de los facilitadores que favorecen una respuesta más ajustada a la diversidad del alumnado. En consecuencia, debe ponerse en valor la pertinencia de iniciar procesos de reflexión en los 
centros educativos que permitan indagar sobre las prácticas que en ellos se realizan, así como reconocer, prevenir y eliminar las posibles barreras existentes en las instituciones escolares.

En este sentido, urge conocer las barreras y los factores que actúan como limitadores de la presencia, la participación y el éxito de todo el alumnado (Echeita, 2017) y que, por tanto, frenan sus procesos de aprendizaje (Forteza, Fuster y Moreno-Tallón, 2019). De igual modo, hay que identificar e impulsar los facilitadores que promueven el desarrollo de una educación más inclusiva (Echeita, Fernández-Blázquez y Simón, 2019; González-Gil et al., 2019).

Este artículo aborda, precisamente, una de las preocupaciones más complejas que en los últimos años viene manifestando la comunidad educativa (profesorado, equipos directivos, familias, alumnado), que no es otra que conocer el verdadero alcance que está teniendo el avance hacia la escuela inclusiva. Una escuela en la que todos los niños y niñas son bienvenidos y encuentran oportunidades para participar, ser valorados y aprender.

La fundamentación teórica que se presenta a continuación trata de alumbrar en qué medida la utilización de las guías que están apareciendo en la literatura como soporte para apoyar el desarrollo de la inclusión en las escuelas puede ayudar en la transformación educativa y social que subyace a este fin. Paralelamente, a lo largo del artículo se reflexiona sobre si estas guías pueden actuar como elementos propulsores (catalizadores) de cambios sustanciales y si cabe esperar que su uso impulse el desarrollo de prácticas educativas orientadas al avance de la inclusión dentro y fuera de las aulas.

\section{2. ¿Se puede medir la inclusión en los centros educativos?}

El Informe de Seguimiento de la Educación en el Mundo para 2020, cuyo leitmotiv son la inclusión y la educación, alude a la importancia de "medir la inclusión en la educación”, con objeto de valorar si los diferentes países están avanzando camino en esta dirección (UNESCO, 2020).

La medición del grado de inclusión se puede y se debe hacer en los centros educativos, aunque ciertamente es un aspecto que no está exento de controversia. Investigaciones previas en este campo han identificado en una misma área geográfica centros poco inclusivos, centros que caminan hacia la inclusión y centros inclusivos (Azorín, 2018). Es importante que los centros educativos hagan una reflexión acerca del estado en el que se encuentra la inclusión en el seno de sus instituciones. Este proceso les permitirá posteriormente elaborar planes de acción contextualizados y abordar las barreras que pueden estar obstaculizando el camino a una educación más inclusiva (Azorín y Ainscow, 2020).

Un aspecto que está empezando a plantearse cada vez con más fuerza desde los diferentes sectores educativos y sociales es en qué medida los centros educativos llevan a cabo la inclusión. De ahí que aparezcan nuevas herramientas e instrumentos para la estimación/valoración de la inclusión. Etxabe, López-Vélez y Soria (2018) en 
su guía Por una escuela que responda a las particularidades del alumnado y reconozca la capacidad de cada persona para aprender proponen un proceso de evaluación de la inclusión mediante un "inclusiómetro". En esta misma línea, Echeita et al. (2019) apuestan por medir la temperatura de la inclusión a través de un “termómetro". Estos medidores ayudan a los centros educativos a obtener información sobre cómo están desarrollando el proceso de inclusión y si necesitan acometer cambios para mejorarlo.

En definitiva, la comunidad educativa debe estar al día de los instrumentos que tiene a su alcance para evaluar, mejorar y avanzar en el ámbito de la educación inclusiva. Es en esta intersección de caminos donde adquiere relevancia el uso de una serie de guías que están a disposición de los centros escolares para aproximarse al estado de la inclusión y para acompañar a los profesionales que se dedican a la enseñanza durante su particular recorrido, no exento de obstáculos, hacia la inclusión.

\section{3. ¿Qué entendemos por "guías” y para qué sirven?}

El concepto educativo de “guías” que proponen Azorín y Sandoval (2019, p. 11) contempla todos aquellos documentos que cuentan con materiales, recursos y herramientas cuya finalidad es "apoyar a las escuelas para ponerse en acción en lo que a cuestiones de inclusión se refiere”.

Generalmente, puede afirmarse que estas guías formulan acciones encaminadas a avanzar en la temática que profundizan. En educación, las guías suelen utilizarse para apoyar acciones, orientar prácticas, acompañar al colectivo al que se dirigen y estimular procesos de formación y mejora. En particular, su uso permite a los centros educativos realizar un análisis pormenorizado de las debilidades/fortalezas sobre el contenido que en ellas se aborda.

Tal y como se expone en este artículo, son numerosas las guías que están dirigidas a la acción educativa y, más concretamente, a la consecución y el avance de la educación inclusiva. Recientemente, se han diseñado multitud de guías que proporcionan pautas para reflexionar y analizar la realidad de la que parten los centros educativos que quieren emprender el desarrollo de prácticas más inclusivas.

Con respecto a los estudios previos que han realizado con anterioridad investigaciones y análisis sobre este tema, cabe destacar: Instrumentos para la autoevaluación y la mejora de la atención a la diversidad en centros educativos (Guirao y Arnaiz, 2014), Revisión de instrumentos sobre atención a la diversidad para una educación inclusiva de calidad (Azorín, Arnaiz y Maquilón, 2017), Algunas propiedades psicométricas de las guías para evaluar prácticas inclusivas en el aula (García, Romero, Escalante y Flores, 2018), Promoviendo la participación del alumnado en la escuela. Análisis de materiales y guias internacionales para docentes que desarrollan experiencias de voz del alumnado (Ceballos y Saiz, 2019) y Apoyos para avanzar hacia una educación más inclusiva en los centros escolares: análisis de guías para la acción (Azorín y Sandoval, 2019).

Con el propósito de continuar la línea de investigación emprendida por los citados estudios previos, en este artículo serán objeto de revisión y análisis otras guías sobre inclusión (distintas a las ya revisadas anteriormente) que están en uso hoy, lo que 
permitirá conocer los aspectos que estos documentos proponen para progresar en la inclusión, por un lado, y para contribuir con un contenido original a este campo de conocimiento, por otro.

\section{Objetivos}

El objetivo principal de este trabajo es llevar a cabo una selección de guías sobre inclusión que resulten de utilidad para iniciar procesos de reflexión-acción en los centros educativos. Durante esta primera fase del estudio, se pondrá especial atención a la finalidad y propósito que tiene la guía, el concepto de inclusión del que parte, el colectivo al que se dirige y la etapa en la que tiene mayor funcionalidad. Asimismo, los objetivos específicos que se formulan a este respecto son:

1. Identificar las barreras que obstaculizan la inclusión y los aspectos facilitadores que la posibilitan. En este sentido, se tendrán en cuenta los principios de presencia, participación y éxito de todo el alumnado.

2. Concretar los recursos/instrumentos que ofrecen las guias para acompañar a los centros educativos en la planificación del viaje hacia una educación más inclusiva. Teniendo en mente este propósito, se va a profundizar en las orientaciones, las estrategias y, más particularmente, en los recursos e instrumentos que aportan las guías.

El siguiente apartado presenta el método utilizado en este estudio.

\section{Método}

Se ha llevado a cabo un estudio de carácter cualitativo sobre una selección de "guías" cuya temática principal es la inclusión. Para ello, se ha hecho uso del análisis documental de contenido (Abela, 2003; Barbosa, Barbosa y Rodríguez, 2013; Bernal, 2006; López, 2002; Rojas, 2011). En el campo de la investigación educativa, este tipo de análisis permite incidir en los aspectos de naturaleza cualitativa que presentan determinadas herramientas, guías o instrumentos. Diferentes estudios respaldan la utilización del análisis documental de contenido para recabar información sobre guías e instrumentos en el campo de la educación inclusiva (Azorín, 2016; Azorín y Sandoval, 2019; Castro, 2017). A este respecto, García-Sanz y Martínez (2012, p. 172) indican que:

Los trabajos documentales pueden definirse como un conjunto de informaciones precisas en relación con un tema determinado que, para poder comunicar una serie de resultados de forma sistemática y organizada, necesitan de un proceso de búsqueda e interpretación exhaustiva de las diversas fuentes documentales.

Partiendo de esta premisa, se expone a continuación: 1) cómo se ha llevado a cabo el proceso de selección de fuentes documentales (guías) atendiendo principalmente al criterio temático escogido; 2) cuáles han sido las guías seleccionadas; 3) cómo se ha rea- 
lizado la construcción del instrumento (hoja/cuadro de registro), y 4) qué procedimiento se ha seguido, haciendo especial incidencia en las diferentes etapas/fases del estudio.

\subsection{Fuentes documentales}

Las fuentes documentales fueron las propias guías seleccionadas para la revisión/ análisis de contenido. Durante la fase de preselección se realizó una adaptación de los criterios ya propuestos anteriormente por Azorín y Sandoval (2019), quedando matizados para este trabajo de la siguiente manera:

a) Guías que anteriormente no han sido analizadas en otros estudios. No se han revisado las guías que ya fueron investigadas en los citados estudios previos, puesto que la intencionalidad es construir un conocimiento original sobre otras guías que se encuentran en uso hoy dentro del ámbito de la inclusión.

b) Temática de las guías vinculada a la inclusión. Esto favorece una aproximación al concepto y significado que particularmente estas guías aportan sobre la inclusión, los facilitadores que potencian/posibilitan el desarrollo de buenas prácticas para atender a la diversidad del alumnado y los recursos que están al alcance de los centros escolares para la valoración de sus prácticas.

c) Actualidad. El intervalo/horquilla de tiempo que se ha recogido en las guías va de 2015 a 2019 (últimos cinco años). Precisamente, esta recopilación ofrece un corpus de guías que incorporan una visión actualizada sobre las tendencias que se consideran útiles para responder a la diversidad en los centros educativos contemporáneos.

d) Visión nacional e internacional. La mayoría de las guías seleccionadas se dirigen a la comunidad de habla hispana. Si bien, conviene aclarar que la revisión realizada por Azorín y Sandoval (2019) contiene un amplio abanico de guías, todas ellas escritas en lengua inglesa, de ahí que se haya optado mayoritariamente por la selección de guías en español, aunque también se incluyen algunas en inglés.

e) Utilidad. Se ha realizado una primera criba escogiendo prioritariamente aquellas guías que incorporan recursos para que los centros educativos puedan valorar y conocer sus puntos de partida a la hora de emprender el camino hacia la inclusión.

f) Finalidad (informativa, formativa y evaluativa). Se ha especificado si se trata de guías que aportan información acerca de cuestiones generales relacionadas con la inclusión; guías que tienen un carácter formativo sobre contenidos asociados a la atención a la diversidad y a la educación inclusiva; y/o guías cuya función es evaluativa, por lo que permiten a los centros escolares desarrollar procesos de evaluación/autoevaluación de prácticas.

La tabla que se muestra a continuación presenta por orden cronológico las guías objeto de estudio (Tabla 1$)$ : 


\begin{tabular}{|c|c|c|c|c|c|}
\hline \multicolumn{6}{|c|}{ TABLA 1. Guias seleccionadas } \\
\hline Guías & Autoría & Organismo & Colectivo & Etapa & Dirección URL \\
\hline $\begin{array}{l}\text { Guia informati- } \\
\text { va para familia- } \\
\text { res de alumnos } \\
\text { y alumnas con } \\
\text { necesidades } \\
\text { educativas } \\
\text { especiales }\end{array}$ & \begin{tabular}{|l|} 
Comisión \\
de Familias \\
en Inclusión \\
de FEAPS \\
$(2015)$
\end{tabular} & $\begin{array}{l}\text { Plena inclu- } \\
\text { sión }\end{array}$ & $\begin{array}{l}\text { Familias y } \\
\text { otros agentes } \\
\text { educativos y } \\
\text { sociales }\end{array}$ & $\begin{array}{l}\text { Infantil, } \\
\text { Primaria y } \\
\text { Secundaria }\end{array}$ & $\begin{array}{l}\text { https://www. } \\
\text { plenainclusion.org/ } \\
\text { sites/default/files/ } \\
\text { guiafamiliaresesco- } \\
\text { lar.pdf }\end{array}$ \\
\hline $\begin{array}{l}\text { A Guide to } \\
\text { Planning an In- } \\
\text { clusion Project }\end{array}$ & \begin{tabular}{|l|} 
Yarbrough, \\
Brandt \\
y Moore \\
$(2016)$
\end{tabular} & $\begin{array}{l}\text { Virginia De- } \\
\text { partment of } \\
\text { Education }\end{array}$ & $\begin{array}{l}\text { *Comunidad } \\
\text { educativa }\end{array}$ & $\begin{array}{l}\text { Primaria, } \\
\text { Secundaria y } \\
\text { Bachillerato }\end{array}$ & $\begin{array}{l}\text { http://www.doe. } \\
\text { virginia.gov/spe- } \\
\text { cial_ed/transition_- } \\
\text { svcs/inclusion- } \\
\text { project.pdf }\end{array}$ \\
\hline $\begin{array}{l}\text { Guia rápida } \\
\text { para promover } \\
\text { la participación } \\
\text { en las escuelas }\end{array}$ & $\begin{array}{l}\text { Ceballos } \\
(2017)\end{array}$ & $\begin{array}{l}\text { Universidad } \\
\text { de Cantabria: } \\
\text { Inclusiónlab }\end{array}$ & $\begin{array}{l}\text { Profesorado } \\
\text { y alumnado }\end{array}$ & $\begin{array}{l}\text { Infantil y } \\
\text { Primaria }\end{array}$ & $\begin{array}{l}\text { https://repo- } \\
\text { sitorio.unican. } \\
\text { es/xmlui/hand- } \\
\text { le/10902/13273 }\end{array}$ \\
\hline $\begin{array}{l}\text { Educación } \\
\text { inclusiva y de } \\
\text { calidad, un de- } \\
\text { recho de todos }\end{array}$ & $\begin{array}{l}\text { Cobeñas et } \\
\text { al. (2017) }\end{array}$ & $\begin{array}{l}\text { COPIDIS y } \\
\text { Grupo Art. } \\
24 \text { por la } \\
\text { Educación } \\
\text { Inclusiva }\end{array}$ & $\begin{array}{l}\text { Comunidad } \\
\text { educativa }\end{array}$ & $\begin{array}{l}\text { Infantil, } \\
\text { Primaria y } \\
\text { Secundaria }\end{array}$ & $\begin{array}{l}\text { https://grupoart24. } \\
\text { org/downloads/ } \\
\text { publicaciones/ma- } \\
\text { nual_educacion_in- } \\
\text { clusiva.pdf }\end{array}$ \\
\hline $\begin{array}{l}\text { El camino hacia } \\
\text { la Educación } \\
\text { Inclusiva. Un } \\
\text { análisis desde la } \\
\text { perspectiva de } \\
\text { los profesionales } \\
\text { de la Educación }\end{array}$ & $\begin{array}{l}\text { Plena inclu- } \\
\text { sión } \\
(2018)\end{array}$ & $\begin{array}{l}\text { Plena Inclu- } \\
\text { sión } \\
\text { Madrid }\end{array}$ & $\begin{array}{l}\text { Comunidad } \\
\text { educativa }\end{array}$ & $\begin{array}{l}\text { Infantil, } \\
\text { Primaria y } \\
\text { Secundaria }\end{array}$ & $\begin{array}{l}\text { https://plenain- } \\
\text { clusionmadrid. } \\
\text { org/wp-content/ } \\
\text { uploads/2018/06/ } \\
\text { CaminoEduInclu- } \\
\text { siva.pdf }\end{array}$ \\
\hline $\begin{array}{l}\text { Escuela inclu- } \\
\text { siva. Por una } \\
\text { escuela que } \\
\text { responda a las } \\
\text { particularidades } \\
\text { del alumnado } \\
\text { y reconozca la } \\
\text { capacidad de } \\
\text { cada persona } \\
\text { para aprender }\end{array}$ & \begin{tabular}{|l|} 
Etxabe et al. \\
$(2018)$
\end{tabular} & $\begin{array}{l}\text { FEVAS Ple- } \\
\text { na Inclusión } \\
\text { Euskadi }\end{array}$ & Profesorado & $\begin{array}{l}\text { Infantil, } \\
\text { Primaria y } \\
\text { Secundaria }\end{array}$ & $\begin{array}{l}\text { https://fevas. } \\
\text { org/?wpfb_dl=146 }\end{array}$ \\
\hline
\end{tabular}




\begin{tabular}{|c|c|c|c|c|c|}
\hline $\begin{array}{l}\text { Guia meto- } \\
\text { dológica para } \\
\text { el tránsito } \\
\text { educativo de } \\
\text { estudiantes con } \\
\text { discapacidad a } \\
\text { la universidad }\end{array}$ & $\begin{array}{l}\text { Medina } \\
\text { y Alcaín } \\
(2018)\end{array}$ & $\begin{array}{l}\text { Fundación } \\
\text { ONCE }\end{array}$ & $\begin{array}{l}\text { Comunidad } \\
\text { educativa }\end{array}$ & $\begin{array}{l}\text { Secundaria, } \\
\text { Bachillerato } \\
\text { y Superior }\end{array}$ & $\begin{array}{l}\text { https://blogs.co- } \\
\text { millas.edu/fei/wp- } \\
\text { content/uploads/ } \\
\text { sites/7/2018/11/ } \\
\text { guia_metodologi- } \\
\text { ca_para_el_transi- } \\
\text { to_educativo_de_- } \\
\text { estudiantes_con_- } \\
\text { discapacidad_a_la_- } \\
\text { universidad_1.pdf }\end{array}$ \\
\hline $\begin{array}{l}\text { Inclusión } \\
\text { y universi- } \\
\text { dad. Guía de } \\
\text { orientaciones, } \\
\text { adaptaciones y } \\
\text { recursos para } \\
\text { la comunidad } \\
\text { universitaria }\end{array}$ & $\begin{array}{l}\text { Bretones, } \\
\text { Flórez y } \\
\text { Rodríguez- } \\
\text { Martín } \\
(2018)\end{array}$ & $\begin{array}{l}\text { Universidad } \\
\text { de Oviedo }\end{array}$ & $\begin{array}{l}\text { Comunidad } \\
\text { educativa }\end{array}$ & $\begin{array}{l}\text { Educación } \\
\text { Superior }\end{array}$ & $\begin{array}{l}\text { https://dptoccedu. } \\
\text { uniovi.es/noti- } \\
\text { cias/-/asset_pu- } \\
\text { blisher/HhG3/ } \\
\text { content/inclusion- } \\
\text { y-universidad-guia- } \\
\text { de-orientaciones- } \\
\text { uniovi;jsessionid= } \\
\text { D8C3AEF90A06 } \\
\text { 688A8944AC7F1 } \\
\text { C6663C3?redirect } \\
\text { =\%2F }\end{array}$ \\
\hline $\begin{array}{l}\text { Implementing } \\
\text { inclusive educa- } \\
\text { tion: a guide for } \\
\text { schools }\end{array}$ & $\begin{array}{l}\text { Knowledge } \\
\text { and Human } \\
\text { Develop- } \\
\text { ment } \\
\text { Authority } \\
(2019)\end{array}$ & KHDA & $\begin{array}{l}\text { Comunidad } \\
\text { educativa }\end{array}$ & \begin{tabular}{|l} 
Infantil, \\
Primaria, \\
Secundaria y \\
Superior
\end{tabular} & $\begin{array}{l}\text { https://islamicedu- } \\
\text { cationbymuqeet. } \\
\text { files.wordpress. } \\
\text { com/2019/01/ } \\
\text { implementing- } \\
\text { inclusive- } \\
\text { education_a-guide- } \\
\text { for-schools_khda. } \\
\text { pdf }\end{array}$ \\
\hline $\begin{array}{l}\text { Implementando } \\
\text { la inclusión en } \\
\text { las escuelas. } \\
\text { Guia sobre } \\
\text { cómo empe- } \\
\text { zar a dirigir e } \\
\text { implementar } \\
\text { vuestro desarro- } \\
\text { llo escolar hacia } \\
\text { la inclusión }\end{array}$ & $\begin{array}{l}\text { Fundación } \\
\text { Plataforma } \\
\text { Educativa } \\
\text { (2019a) }\end{array}$ & $\begin{array}{l}\text { Consorcio } \\
\text { del proyecto } \\
\text { IIIS }\end{array}$ & $\begin{array}{l}\text { Comunidad } \\
\text { educativa }\end{array}$ & $\begin{array}{l}\text { Primaria y } \\
\text { Secundaria }\end{array}$ & $\begin{array}{l}\text { https://cdn. } \\
\text { website-editor.net/ } \\
7719483231 \text { fe4883 } \\
\text { a9297404e957bcce/ } \\
\text { files/uploaded/Gui } \\
\text { a\%2520IIIS\%2520 } \\
\text { Espa\%25C3\%25B } \\
\text { 1ol.pdf }\end{array}$ \\
\hline
\end{tabular}


CECILIA AZORÍN ABELLÁN Y MÓNICA DEL MAR GONZÁLEZ BOTÍA

\begin{tabular}{|c|c|c|c|c|c|}
\hline $\begin{array}{l}\text { Implementando } \\
\text { la inclusión en } \\
\text { las escuelas. } \\
\text { Módulos de } \\
\text { Capacitación } \\
\text { para apoyar el } \\
\text { desarrollo esco- } \\
\text { lar inclusivo }\end{array}$ & $\begin{array}{l}\text { Fundación } \\
\text { Plataforma } \\
\text { Educativa } \\
\text { (2019b) }\end{array}$ & $\begin{array}{l}\text { Consorcio } \\
\text { del proyecto } \\
\text { IIIS }\end{array}$ & Profesorado & $\begin{array}{l}\text { Primaria y } \\
\text { Secundaria }\end{array}$ & $\begin{array}{l}\text { https://cdn.website-edi } \\
\text { tor.net/7719483231fe48 } \\
\text { 83a9297404e957b- } \\
\text { cce/files/uploaded/ } \\
\text { Training\%2520Mod\% } \\
\text { 2520espv5.pdf }\end{array}$ \\
\hline $\begin{array}{l}\text { La ruta hacia la } \\
\text { escuela inclusi- } \\
\text { va. Avanzando } \\
\text { con la transfor- } \\
\text { mación escolar } \\
\text { desde adentro }\end{array}$ & $\begin{array}{l}\text { Porter y } \\
\text { Towell } \\
(2019)\end{array}$ & $\begin{array}{l}\text { Inclusive } \\
\text { Education } \\
\text { Canada }\end{array}$ & $\begin{array}{l}\text { Comunidad } \\
\text { educativa }\end{array}$ & $\begin{array}{l}\text { Infantil, } \\
\text { Primaria y } \\
\text { Secundaria }\end{array}$ & $\begin{array}{l}\text { https://www. } \\
\text { centreforwelfarere- } \\
\text { form.org/uploads/ } \\
\text { attachment/662/ } \\
\text { la-ruta-hacia-la- } \\
\text { escuela-inclusiva. } \\
\text { pdf }\end{array}$ \\
\hline $\begin{array}{l}\text { Termóme- } \\
\text { tro para la } \\
\text { valoración de } \\
\text { la educación } \\
\text { inclusiva en un } \\
\text { centro escolar. } \\
\text { Programa Red } \\
\text { para la Educa- } \\
\text { ción Inclusiva }\end{array}$ & $\begin{array}{l}\text { Echeita et } \\
\text { al. (2019) }\end{array}$ & $\begin{array}{l}\text { Plena inclu- } \\
\text { sión }\end{array}$ & Profesorado & $\begin{array}{l}\text { Primaria y } \\
\text { Secundaria }\end{array}$ & $\begin{array}{l}\text { https://www. } \\
\text { plenainclusion.org/ } \\
\text { sites/default/files/ } \\
\text { termometro-edu. } \\
\text { pdf }\end{array}$ \\
\hline
\end{tabular}

"El concepto de "comunidad educativa" al que se refiere esta clasificación engloba al profesorado, al alumnado, a las familias y a otros agentes educativos y sociales.

En el siguiente apartado se hace alusión al instrumento elaborado para la recogida de información de las guías.

\subsection{Instrumento}

Se ha construido una boja/cuadro de registro (Tabla 2) que ha permitido recopilar información de las fuentes documentales (guías), considerando los siguientes aspectos: 


\section{TABLA 2. Cuadro de registro}

\begin{tabular}{|c|c|}
\hline Finalidad de la guía & $\begin{array}{l}\text { En este apartado se indica si la finalidad de la guía es for- } \\
\text { mativa, informativa y/o evaluativa. }\end{array}$ \\
\hline Objetivo & Aquí se recoge el propósito por el que se crea la guía. \\
\hline $\begin{array}{l}\text { Concepto de inclusión } \\
\text { que plantea }\end{array}$ & $\begin{array}{l}\text { El concepto de inclusión tiene diferentes acepciones y es } \\
\text { por ello que resulta interesante conocer la postura que } \\
\text { cada guía ofrece a este respecto. }\end{array}$ \\
\hline $\begin{array}{l}\text { Colectivo para el que } \\
\text { se crea }\end{array}$ & $\begin{array}{l}\text { Entre los posibles colectivos destacan el profesorado, el } \\
\text { alumnado, las familias y la comunidad educativa en gene- } \\
\text { ral. El concepto de "comunidad educativa" al que se refie- } \\
\text { re esta clasificación engloba al profesorado, al alumnado, a } \\
\text { las familias y a otros agentes educativos y sociales. }\end{array}$ \\
\hline $\begin{array}{l}\text { Etapa escolar a la que } \\
\text { se dirige }\end{array}$ & $\begin{array}{l}\text { Hace referencia al nivel educativo donde tiene mayor fun- } \\
\text { cionalidad la guía: Educación Infantil, Primaria, Secunda- } \\
\text { ria y/o Superior. }\end{array}$ \\
\hline $\begin{array}{l}\text { Barreras/limitadores } \\
\text { que obstaculizan la } \\
\text { inclusión }\end{array}$ & $\begin{array}{l}\text { Se analiza el conjunto de factores que entorpecen/impi- } \\
\text { den/limitan el establecimiento de prácticas inclusivas des- } \\
\text { de el punto de vista de la guía. }\end{array}$ \\
\hline $\begin{array}{l}\text { Facilitadores que po- } \\
\text { tencian y favorecen el } \\
\text { desarrollo de prácticas } \\
\text { inclusivas }\end{array}$ & $\begin{array}{l}\text { Se exponen las condiciones y prácticas que promueven las } \\
\text { guías para mejorar el proceso de inclusión en los centros. }\end{array}$ \\
\hline \begin{tabular}{|l|} 
Presencia, participación \\
y logro (éxito escolar)
\end{tabular} & $\begin{array}{l}\text { En esta parte se concreta si las guías establecen estas tres } \\
\text { dimensiones (presencia, participación y logro) como prio- } \\
\text { ridades para la materialización de una verdadera educación } \\
\text { inclusiva. }\end{array}$ \\
\hline $\begin{array}{l}\text { Orientaciones que pro- } \\
\text { pone la guí para avan- } \\
\text { zar hacia una educación } \\
\text { más inclusiva }\end{array}$ & $\begin{array}{l}\text { Se revisan las propuestas que aporta cada guía en el acom- } \\
\text { pañamiento y la orientación hacia una mayor inclusión en } \\
\text { los centros educativos. Se expone un breve resumen de los } \\
\text { planes de acción, intervenciones, estrategias o recomenda- } \\
\text { ciones a los que cada guía hace mención. }\end{array}$ \\
\hline \begin{tabular}{|l|} 
Elementos/ingredien- \\
tes/equipaje clave en \\
materia de inclusión so- \\
bre los que se reflexiona \\
\end{tabular} & $\begin{array}{l}\text { Se identifican los elementos que en todas las guías son co- } \\
\text { munes y que no pueden faltar en la maleta cuando hace- } \\
\text { mos el equipaje para emprender el denominado "viaje a la } \\
\text { inclusión". }\end{array}$ \\
\hline $\begin{array}{l}\text { Recursos/instrumentos } \\
\text { que aporta para la revi- } \\
\text { sión práctica }\end{array}$ & $\begin{array}{l}\text { En este apartado se realiza una recogida de los instrumen- } \\
\text { tos/materiales/recursos que las guías aportan para valorar/ } \\
\text { medir/provocar una reflexión sobre el estado de la inclu- } \\
\text { sión en los centros educativos. }\end{array}$ \\
\hline
\end{tabular}

Seguidamente, se expone una breve síntesis sobre las diferentes fases acometidas en este estudio. 


\subsection{Procedimiento}

En este apartado se explica con mayor detalle el proceso seguido para la selección de las guías, así como los aspectos relacionados con la procedencia de estos documentos.

Fase 1. Búsqueda de fuentes documentales. En esta primera fase se establecieron los criterios para la selección de las guías (a los que se hace mención en el apartado de "Fuentes documentales”), lo que permitió determinar los parámetros a tener en cuenta para la estimación/desestimación de las mismas. Esta búsqueda de información se realizó en las siguientes bases de datos: Dialnet, Researchgate, SciELO y Google Académico. Los descriptores o palabras clave fueron: "guías inclusión”, "guías educación inclusiva” y "guías atención diversidad” en español e inglés. Además, se realizó una búsqueda pormenorizada en diferentes portales web que pertenecen a organizaciones como Plena inclusión (https://www.plenainclusion.org), la ONCE (https://www. once.es) y el Consorcio de Educación Inclusiva (https://www.consorcio-educacioninclusiva.es), en los que se hallaron interesantes materiales en línea asociados con la temática objeto de estudio. La Tabla 1 pone a disposición del lector las direcciones web donde se alojan las diferentes guías para su consulta y descarga.

Fase 2. Uso del cuadro de registro para determinar la estimación/desestimación de guias (fuentes documentales). En esta segunda etapa se escogieron aquellas guías que mejor se ajustaban a los criterios establecidos con anterioridad.

Fase 3. En esta última fase, una vez delimitadas las guías para la revisión, se procedió al análisis documental de contenido y se dio respuesta a los objetivos específicos planteados al inicio. Finalmente, se procedió a la identificación de las barreras (limitadores) y de los aspectos facilitadores que se proponen a modo de respuesta, así como a la concreción de los recursos que están a disposición de los centros educativos para la valoración de la inclusión.

\section{Resultados}

A continuación, se presentan los resultados atendiendo a los objetivos formulados al inicio:

- Objetivo principal. Llevar a cabo una selección de guias sobre inclusión que resulten de utilidad para iniciar procesos de reflexión-acción en los centros educativos.

Este artículo gira en torno a la selección de un total de 13 guías. En líneas generales, todas ellas apuntan a la necesidad de poner en marcha procesos de reflexiónacción en los centros educativos que permitan conocer su punto de partida en lo que a términos de inclusión se refiere.

Con respecto a las finalidades, en su mayoría, se trata de guías formativas que aportan propuestas de formación para el desarrollo de escuelas más inclusivas (Etxabe et al., 2018; Fundación Plataforma Educativa, 2019a y b; Knowledge and Human Development Authority, 2019; Medina y Alcain, 2018; Plena inclusión, 2018; Porter y Towell, 2019). Si bien, hay dos guías de naturaleza informativa (Cobeñas et al., 2017; 
Comisión de Familias en Inclusión de FEAPS, 2015) que incorporan un amplio marco teórico e informan al lector acerca de la inclusión en el sistema educativo actual. Además, se ha comprobado que la guía elaborada por Bretones et al. (2018) tiene una doble finalidad (informativa y formativa), pues contiene adaptaciones y recursos para profundizar/reflexionar sobre las diferentes casuísticas que presentan los estudiantes. Por último, hay tres guías cuya finalidad es evaluativa (Ceballos, 2017; Echeita et al., 2019; Yarbrough et al., 2016). Estos documentos centran la atención en cómo se pueden mejorar las escuelas para hacer que sean más inclusivas mediante el uso de recursos/instrumentos para la evaluación/autoevaluación de prácticas.

En relación con el objetivo/propósito que tienen las guías, puede afirmarse que la mayoría pretenden acompañar/guiar a la comunidad educativa en su camino hacia la inclusión, fomentando prácticas y acciones tendentes a generar una mejora. Particularmente, las guías de Etxabe et al. (2018) y Fundación Plataforma Educativa (2019b) tienen como objetivo común fomentar una actitud positiva del profesorado hacia la inclusión. Lo mismo ocurre con las guías de Ceballos (2017) y Medina y Alcain (2018), que coinciden en aumentar la participación de los estudiantes y ponen especial atención en la eliminación de los procesos de exclusión del alumnado con discapacidad. El resto de guías tienen objetivos distintos, entre ellos: reflexionar sobre el importante papel que desempeñan las familias en el campo de la inclusión y describir aspectos generales de la escuela inclusiva (Comisión de Familias en Inclusión de FEAPS, 2015); mostrar experiencias (buenas prácticas) de centros educativos que desarrollan proyectos inclusivos (Fundación Plataforma Educativa, 2019a); así como aportar instrumentos para valorar la inclusión y crear una red de alianzas entre centros (Echeita et al., 2019).

Del análisis de la información se desprende que hay un conjunto de aspectos comunes sobre el concepto de inclusión en la mayoría de las guías revisadas: la respuesta a la diversidad del alumnado, debe darse una respuesta a las necesidades de aprendizaje de todos los estudiantes. Para ello, es imprescindible contar con los medios y el apoyo necesarios; la inclusión como lucha contra la exclusión, la sociedad precisa de un cambio de paradigma para que sea más justa y sin discriminaciones. La inclusión abandera una educación para todas y todos, con especial énfasis en las personas que son más vulnerables. Dentro de esta categoría, la clasificación y el etiquetado se encuentran en el punto de mira; la igualdad de condiciones hacia el aprendizaje, se debe ajustar el aprendizaje a las necesidades, los intereses y las capacidades del alumnado. Esto ha de hacerse de forma equitativa para que todas y todos cuenten con las mismas oportunidades; la participación del alumnado, es importante que el alumnado participe en su proceso de aprendizaje. En este sentido, los fundamentos que sostienen esta participación tienen que ver con el acceso/presencia y el aprendizaje/logro de los estudiantes; proceso de transformación de la escuela, se tiene que dar una reforma en el sistema que promueva el cambio estructural para garantizar el proceso hacia la inclusión; derecho a una educación que verdaderamente incluya a todo el alumnado, la inclusión es un derecho. Derecho a tener las mismas oportunidades. La escuela requiere de una transformación para que la educación que llegue a todo el alumnado sea equitativa. Esta afirmación se apoya en el principio de “no dejar a nadie atrás” 
que sustenta el pensamiento y la práctica de la Agenda 2030; y educación de calidad, que la enseñanza favorezca aprendizajes relevantes, pertinentes y adecuados para una formación integral. Se considera como garante de dicha calidad el máximo desarrollo de las potencialidades del alumnado, independientemente de sus puntos de partida.

En relación con el colectivo al que se dirigen las guías predomina en la mayoría la comunidad educativa (profesorado, alumnado, familias y otros agentes educativos y sociales). Asimismo, las guías de Echeita et al. (2019), Etxabe et al. (2018) y Fundación Plataforma Educativa (2019b) van dirigidas al profesorado; la guía de Ceballos (2017) se dirige al alumnado; y la guía de la Comisión de Familias en Inclusión de FEAPS (2015) está orientada a las familias y a otros agentes educativos y sociales.

Sobre la etapa educativa donde tienen mayor funcionalidad las guías, cinco de ellas están contextualizadas en las etapas de Educación Infantil, Educación Primaria y Educación Secundaria (Cobeñas et al., 2017; Comisión de Familias en Inclusión de FEAPS, 2015; Etxabe et al., 2018; Plena inclusión, 2018; Porter y Towell, 2019) y cuatro para la etapa de Educación Primaria y Educación Secundaria (Echeita et al., 2019; Fundación Plataforma Educativa, 2019a y b; Yarbrough et al., 2016). Las guías de Echeita et al. (2019) y Yarbrough et al. (2016) introducen la etapa de Bachillerato; la guía de Ceballos (2017) va dirigida a las etapas de Educación Infantil y Educación Primaria; la guía de Medina y Alcain (2018) se ha creado para las etapas de Educación Secundaria, Bachillerato y Educación Superior; y la guía de Bretones et al. (2018) para la etapa de Educación Superior. Por último, hay una guía que se dirige a todas las etapas (Knowledge and Human Development Authority, 2019).

Se presentan seguidamente los resultados derivados de los objetivos específicos.

- Objetivo específico 1. Identificar las barreras que obstaculizan la inclusión y los aspectos facilitadores que la posibilitan.

Existe todo un conjunto de barreras que obstaculizan el logro de la inclusión, así como de facilitadores que posibilitan el desarrollo de prácticas más inclusivas en los centros educativos. La tabla que se presenta a continuación incluye una recopilación de los obstáculos más representativos que aparecen en las guías y de las respuestas facilitadoras que se formulan para cada una de estas barreras (Tabla 3):

\section{TABLA 3. Barreras y facilitadores}

\begin{tabular}{|l|l|}
\hline BARRERAS & FACILITADORES \\
\hline $\begin{array}{l}\text { La falta de recursos, } \\
\text { personal y formación }\end{array}$ & $\begin{array}{l}\text { Algunos elementos facilitadores a este respecto tienen que ver con } \\
\text { la capacitación del profesorado en metodologías que favorezcan la } \\
\text { inclusión, el incremento de su formación y un mayor ajuste de los } \\
\text { recursos que están a disposición del profesorado para trabajar con } \\
\text { los diferentes ritmos de aprendizaje. Precisamente, las guías insisten } \\
\text { en la relevancia de construir apoyos y propiciar una acción de coor- } \\
\text { dinación, planificación y seguimiento de las actuaciones que se ponen } \\
\text { en marcha desde el centro escolar. }\end{array}$ \\
\hline
\end{tabular}




\begin{tabular}{|c|c|}
\hline $\begin{array}{l}\text { No bay una cultura } \\
\text { de trabajo colabo- } \\
\text { rativa entre el pro- } \\
\text { fesorado ni redes de } \\
\text { colaboración entre } \\
\text { centros educativos }\end{array}$ & $\begin{array}{l}\text { Las guías analizadas insisten en la necesidad de promover redes de } \\
\text { colaboración con otros profesionales y agentes externos. Estrechar } \\
\text { relaciones entre el personal docente y desarrollar procesos de colabo- } \\
\text { ración, coplanificación y coenseñanza son algunas de las propuestas } \\
\text { facilitadoras que barajan estos documentos. }\end{array}$ \\
\hline $\begin{array}{l}\text { No se cumple el prin- } \\
\text { cipio de accesibilidad } \\
\text { universal }\end{array}$ & $\begin{array}{l}\text { Es importante crear espacios educativos que favorezcan ambientes } \\
\text { acogedores donde se potencien e intercambien experiencias de en- } \\
\text { riquecimiento. Estos espacios tienen que ser accesibles para todas y } \\
\text { todos, creando entornos educativos inclusivos, aspecto que evidente- } \\
\text { mente facilitará una mayor inclusión. }\end{array}$ \\
\hline $\begin{array}{l}\text { Barreras a las que se } \\
\text { enfrenta el alumna- } \\
\text { do con necesidades } \\
\text { educativas especiales } \\
\text { en las aulas }\end{array}$ & $\begin{array}{l}\text { Algunos de los elementos facilitadores que proponen las guías son } \\
\text { los siguientes: agrupamientos heterogéneos; cooperación, apoyo e } \\
\text { igualdad de oportunidades; un currículo más abierto y flexible; la } \\
\text { participación del alumnado en la toma de decisiones educativas; la } \\
\text { tutorización y el desarrollo de habilidades personales. }\end{array}$ \\
\hline $\begin{array}{l}\text { Falta de conciencia } \\
\text { y valoración de la } \\
\text { diversidad, que se } \\
\text { materializa en actos } \\
\text { de discriminación, } \\
\text { segregación y fracaso } \\
\text { escolar }\end{array}$ & $\begin{array}{l}\text { Las guías apuestan por contrarrestar estas limitaciones a través de la } \\
\text { formación, información y comprensión sobre los desafíos a los que } \\
\text { se enfrentan los colectivos más vulnerables. Es necesario que la co- } \\
\text { munidad educativa aprecie las diferencias, cree un clima de respeto, } \\
\text { promueva un sentimiento de empatía, entendimiento y aprendizaje } \\
\text { conjunto. El fin último es que todas las personas se sientan valoradas, } \\
\text { respetadas y reconocidas. }\end{array}$ \\
\hline $\begin{array}{l}\text { Escasa relación y } \\
\text { colaboración entre } \\
\text { familia-centro }\end{array}$ & $\begin{array}{l}\text { La coordinación y colaboración entre familia-escuela es vital para la } \\
\text { eficiente transmisión de la información del alumnado. En este senti- } \\
\text { do, es importante que la familia se implique en los procedimientos de } \\
\text { enseñanza, aprendizaje y evaluación de sus hijas e hijos. Además, los } \\
\text { centros educativos (los profesionales que trabajan en ellos) tienen que } \\
\text { brindar nexos de unión y construir puentes para la comunicación y la } \\
\text { colaboración con las familias. Los padres y las madres deben disponer } \\
\text { de oportunidades que les permitan formar parte del día a día de los } \\
\text { centros escolares e involucrarse en ellos de una manera activa. }\end{array}$ \\
\hline $\begin{array}{l}\text { No se escucha la voz } \\
\text { del alumnado }\end{array}$ & $\begin{array}{l}\text { a potenciar el diálogo } \\
\text { que los estudiantes se }\end{array}$ \\
\hline $\begin{array}{l}\text { Problemas v } \\
\text { dos con el trán. }\end{array}$ & rom \\
\hline
\end{tabular}




\begin{tabular}{|l|l|}
\hline $\begin{array}{l}\text { Normativa } \\
\text { desactualizada }\end{array}$ & $\begin{array}{l}\text { Se necesita poner en marcha políticas educativas que se ajusten a ne- } \\
\text { cesidades de la escuela del siglo XXI. Algunas guías exponen las polí- } \\
\text { ticas. Esto implica un mayor esfuerzo e incremento de recursos para } \\
\text { que el sistema educativo pueda mejorar la atención y la respuesta a la } \\
\text { diversidad de necesidades/metas del alumnado. }\end{array}$ \\
\hline $\begin{array}{l}\text { Etiquetaje propio del } \\
\text { modelo clinico }\end{array}$ & $\begin{array}{l}\text { Entre los facilitadores que proponen las guías a este respecto destaca } \\
\text { la eliminación del diagnóstico para no etiquetar a los estudiantes y la } \\
\text { apuesta por un proceso de evaluación más inclusivo. }\end{array}$ \\
\hline $\begin{array}{l}\text { Sociedad con prejui- } \\
\text { cios y rechazo a la } \\
\text { discapacidad }\end{array}$ & $\begin{array}{l}\text { El principal facilitador que se plantea para lograr un cambio a este } \\
\text { respecto es sensibilizar e implicar a toda la sociedad para aprender } \\
\text { a celebrar la diferencia como rasgo propio de la diversidad humana. }\end{array}$ \\
\hline $\begin{array}{l}\text { Incumplimiento del } \\
\text { derecho a la inclusión }\end{array}$ & $\begin{array}{l}\text { El elemento facilitador aquí es el reconocimiento del derecho de to- } \\
\text { dos los estudiantes a acceder a una educación de calidad, eliminando } \\
\text { los procesos excluyentes que se producen por motivos que se relacio- } \\
\text { nan con la diversidad de las personas. }\end{array}$ \\
\hline
\end{tabular}

Por último, doce de las trece guías analizadas relacionan la inclusión con los principios de presencia, participación y logro/éxito escolar. A este respecto, tanto las barreras como los facilitadores giran en torno a estos principios, que son fundamentales para que exista una verdadera inclusión educativa. Particularmente, la guía elaborada por Porter y Towell (2019) concreta que la escuela necesita poner en práctica sistemas y medidas apropiadas para monitorear (realizar un seguimiento) la presencia, la participación y el progreso de todos los estudiantes.

- Objetivo específico 2. Concretar los recursos/instrumentos que ofrecen las guías para acompañar a los centros educativos en la planificación del viaje hacia una educación más inclusiva.

Cada guía tiene su propia idiosincrasia y fórmula para la educación inclusiva. Entre las propuestas más destacadas se encuentran las siguientes: promover proyectos y planes de acción para la inclusión; ofrecer diferentes estrategias inclusivas para la implementación en el aula/centro; y recomendar recursos de utilidad, así como orientaciones para el desarrollo de prácticas de naturaleza inclusiva.

Hay guías que aportan instrumentos para la evaluación/valoración de prácticas en los centros educativos. Concretamente, la guía elaborada por Yarbrough et al. (2016) ofrece una evaluación pretest/postest, dos encuestas y un formulario. La guía desarrollada por Ceballos (2017) contiene un mapa conceptual con una serie de preguntas para que los centros midan su puesta en marcha; y la guía creada por Echeita et al. (2019) aporta un "termómetro" para medir la temperatura de la inclusión e identificar los ámbitos en los que necesitan mejorar los centros educativos.

Sin embargo, otras guías no ofrecen instrumentos para la valoración de prácticas, aunque debe reconocerse que la guía propuesta por la Comisión de Familias en Inclusión de FEAPS (2015) sí proporciona recomendaciones y orientaciones que pueden 
resultar útiles para la intervención. Además, la guía desarrollada por Bretones et al. (2018) contiene recursos y enlaces de interés que indudablemente pueden resultar útiles a la hora de plantearse procesos de autoevaluación de prácticas.

El resto de guías analizadas no disponen de instrumentos de valoración. La guía de la Fundación Plataforma Educativa (2019b) utiliza módulos de capacitación como recursos para la formación del profesorado; al igual que la guía elaborada por la Knowledge and Human Development Authority (2019), que aporta una serie de medidas que las escuelas pueden adoptar como recursos para reducir la discriminación. En esta línea, se establecen también consideraciones finales a tener en cuenta (Bretones et al., 2018); reflexiones/preguntas (Etxabe et al., 2018); y ejemplos prácticos de profesionales que intervienen en el día a día de los centros educativos (Fundación Plataforma Educativa, 2019a; Medina y Alcain, 2018; Plena inclusión, 2018; Porter y Towell, 2019).

\section{Discusión y conclusiones}

Este artículo presenta una selección de guías sobre inclusión que posibilitan el inicio de procesos de reflexión-acción en los centros educativos. Debe reconocerse el potencial que tienen estas guías, en tanto instrumentos que favorecen la autoevaluación de los centros educativos y la puesta en marcha de procesos de cambio y mejora (Arnaiz, De Haro y Azorín, 2018; Guirao y Arnaiz, 2014).

En términos generales, el objetivo/propósito que tienen las guías es el de orientar/ guiar/acompañar a la comunidad educativa en el viaje hacia la inclusión, favoreciendo procesos de apoyo y asesoramiento en sus prácticas.

En relación con el concepto de inclusión, grosso modo, la mayoría de las guías coinciden con la definición que aporta Ainscow (2015) como proceso de lucha contra la exclusión. Los centros educativos tienen que reformular sus prácticas y acciones para mejorar la atención a la diversidad del alumnado siguiendo los fundamentos de la inclusión. En esta línea, todas las guías analizadas abordan la definición de inclusión permitiendo al lector construir una idea detallada de lo que significa este concepto. Del conjunto de guías se puede extraer que el término más generalizado se refiere al que integra y reconoce a la diversidad del alumnado teniendo en cuenta las características propias de cada persona, eliminando los procesos excluyentes y ofreciendo de forma equitativa las mismas oportunidades hacia el aprendizaje. Adicionalmente, las guías analizadas ponen de manifiesto la importancia de la participación del alumnado y la necesidad de que se produzcan cambios y reformas en las políticas, culturas y prácticas, tal como indica el propio Index for Inclusion de Booth y Ainscow (2011).

Por otro lado, la mayoría de las guías han sido diseñadas para su uso por parte de toda la comunidad educativa, por lo que se remarca el decisivo rol que tienen los diferentes agentes para la transformación del cambio hacia la inclusión. Es importante que todos se involucren y que adquieran el compromiso e implicación que se necesita para progresar hacia un sistema verdaderamente inclusivo (Ainscow, 2017). 
En lo que respecta a las etapas educativas, se han hallado semejanzas con la investigación realizada por Azorín y Sandoval (2019). En ambos trabajos, la mayoría de las guías se dirigen a las etapas de Educación Primaria y de Educación Secundaria, quedando en un segundo plano las etapas de Educación Infantil y de Educación Superior.

Desde el punto de vista de los facilitadores, las guías recogen la relevancia que tienen las redes de colaboración por parte de los diferentes miembros de la comunidad educativa, así como la importancia del liderazgo distribuido, lo que coincide con investigaciones recientes que ponen en alza estas nuevas tendencias en el campo de la educación (Azorín, Harris y Jones, 2020). En los últimos años, la literatura especializada en la inclusión ha destacado que las redes de colaboración juegan un papel determinante (Azorín, 2020b; Azorín y Arnaiz, 2018). De hecho, cada vez son más los centros educativos que crean redes de apoyo y colaboración para la mejora de la educación inclusiva (Arnaiz et al., 2018). En suma, las redes son cruciales para apoyar prácticas inclusivas que permitan hacer frente al desafío de la diversidad (Harris y Jones, 2020; UNESCO, 2020).

Echeita (2013) insiste en la importancia de identificar las barreras para focalizar los esfuerzos y convertir las debilidades en fortalezas. Precisamente, las guías tienen un gran potencial como elementos de apoyo en los centros educativos. Con respecto a la utilidad de las guías, puede afirmarse que:

- La planificación del camino hacia una educación más inclusiva requiere de una serie de orientaciones para avanzar en esta dirección. Las diferentes guías revisadas presentan un conjunto de propuestas de acompañamiento que incorporan acciones, estrategias, planes, proyectos y orientaciones concretas para la eliminación de los procesos excluyentes en los centros educativos.

- Las guias aportan recursos/instrumentos que facilitan la evaluación de la inclusión. Estos instrumentos/materiales/recursos adoptan forma de cuestionarios, escalas, rúbricas, entrevistas, grupos de discusión y preguntas de reflexión, entre otros. Todos ellos favorecen la puesta en marcha de procesos de autoevaluación; actúan como recursos para la mejora de la atención a la diversidad; generan una mayor comprensión y escucha de los diferentes colectivos; evalúan prácticas inclusivas del profesorado dentro y fuera de las aulas, y apoyan a los centros escolares en el desarrollo de prácticas más inclusivas.

- Los profesionales de los centros educativos desconocen el importante apoyo que pueden brindar los recursos y guías que en este trabajo se han analizado y, en ocasiones, carecen de la suficiente formación e información para responder de manera inclusiva a la diversidad del alumnado. En síntesis, el conocimiento de estos recursos facilita al profesorado la posibilidad de formarse autónomamente y brinda a los equipos directivos pautas acerca de cómo hacer que sus centros educativos sean más inclusivos (Ceballos y Saiz, 2019).

Resulta, pues, esencial contar con herramientas y estrategias que permitan acometer una enseñanza más eficaz e inclusiva. Por tanto, la utilización de estas guías en los centros educativos es, sin lugar a dudas, un aspecto que marca la diferencia.

Unido a ello, hay que reconocer que de poco o nada sirven las guías si finalmente estos documentos acaban ocupando un espacio más en las estanterías de los centros 
educativos o en los repositorios de información digital. Para que estos documentos sean efectivos, se necesita de un equipo humano comprometido con la atención a la diversidad, con capacidad de autocrítica y con ganas de mejorar. Profesionales preparados para mirarse en el espejo educativo y emprender procesos de reflexión-acción.

Más allá de los debates en torno a las diferencias humanas, dentro de las generaciones actuales y futuras, hay toda una infancia y una juventud que espera a ser atendida, visibilizada y correspondida como merece. La inclusión impuesta desde instancias externas nunca funcionará. En realidad, cabría preguntarse si los profesionales de la educación están preparados para acometer el desafío de la diversidad y proporcionar una educación inclusiva, sin excepciones. Una educación que vaya más allá de la mentalidad propia del estigma y del etiquetaje, que salvaguarde simplemente el derecho a la inclusión y emane de una voluntad firme de cambio (UNESCO, 2020).

Finalmente, este artículo resulta útil en tanto que puede ayudar a los profesionales de la educación y de la orientación escolar a discernir y escoger la guía que mejor se adapte a sus intereses y necesidades. Es importante que los centros y, en definitiva, las personas que trabajan en ellos, se embarquen en procesos de cambio e innovación queles ayuden a mejorar sus prácticas educativas (Azorín, 2020a). La plena inclusión que lleva décadas reclamándose en España, y en otros países del mundo, inexorablementellegará cuando se empodere a toda la comunidad educativa para conquistar este logro.

A modo de cierre, puede concluirse que la oportunidad que otorgan las guías para repensar otra forma de hacer educación genera la sinergia que se precisa para que el cambio hacia la inclusión sea posible.

\section{Referencias bibliográficas}

Abela, J. (2003). Las técnicas de Análisis de Contenido: una revisión actualizada. Recuperado de http://mastor.cl/blog/wp-content/uploads/2018/02/Andreu.-analisis-de-contenido.34-pags-pdf.pdf

Ainscow, M. (2015). Struggling for equity in education: the legacy of Salamanca. En F. KiUppis y R. S. Hausstätter (Eds.), Inclusive education twenty years after Salamanca (pp. 41-55). New York: Peter Lang.

Ainscow, M. (2017). Pasos para la inclusión en las escuelas. Recuperado de http://www.eduforics.com/es/pasos-la-inclusion-las-escuelas/

Ainscow, M. (2020). Promoting inclusion and equity in education: lessons from international experiences. Nordic Journal of Studies in Educational Policy, 6(1), 7-16. doi: 10.1080/20020317.2020.1729587

Arnaiz, P., De Haro, R. y Azorín, C. (2018). Redes de apoyo y colaboración para la mejora de la educación inclusiva. Profesorado. Revista de Currículum y Formación de Profesorado, 22(2), 29-49. doi: 10.30827/profesoradov22i2.7713

Azorín, C. (2016). La respuesta a la diversidad del alumnado en el contexto inglés: un estudio de casos. Enseñanza and Teaching, 34(2), 77-91. doi: 10.14201/et20163427791

Azorín, C. (2018). The journey towards inclusion: exploring the response of teachers to the challenge of diversity in schools. Revista Colombiana de Educación, 75, 39-58. 
Azorín, C. (2020a). Beyond COVID-19 supernova. Is another education coming? Journal of Professional Capital and Community, 5(3). doi: 10.1108/JPCC-05-2020-0019

Azorín, C. (2020b). Leading networks. School Leadership and Management, 40(2-3), 105-110. https://doi.org/10.1080/13632434.2020.1745396

Azorín, C. y Ainscow, M. (2020). Guiding schools on their journey towards inclusión. International Journal of Inclusive Education, 24(1), 58-76. doi: 10.1080/13603116.2018.14500900

Azorín C. y Arnaiz, P. (2018). Editorial: Redes de Colaboración en educación. Nuevas formas de participación y transformación social. Revista de Currículum y Formación del Profesorado, 22(2), 1-6. doi: 10.30827/profesorado.v22i2.7846

Azorín, C., Arnaiz, P. y Maquilón, J. J. (2017). Revisión de instrumentos sobre atención a la diversidad para una educación inclusiva de calidad. Revista Mexicana de Investigación Educativa, 22(75), 1021-1045.

Azorín, C., Harris, A. y Jones, M. (2020). Taking a distributed perspective on leading professional learning networks. School Leadership and Management, 40(2-3), 111-127. doi: 10.1080/13632434.2019.1647418

Azorín, C. y SAndoval, M. (2019). Apoyos para avanzar hacia una educación más inclusiva en los centros escolares: análisis de guías para la acción. Siglo Cero, 50(3), 7. doi: 10.14201/ scero2019503727

Barbosa, J. W., Barbosa, J. C. y Rodríguez, M. (2013). Revisión y análisis documental para el estado del arte: una propuesta metodológica desde el contexto de la sistematización de experiencias educativas. Investigación Bibliotecológica: Archivología, Bibliotecología e Información, 27(61), 83-105. doi: 10.1016/s0187-358x(13)72555-3

Bernal, C. A. (2006). Metodología de la investigación para administración, economía, humanidades y ciencias sociales. Naucalpan: Pearson Educación.

Воотн, T. у Ainscow, M. (2011). Index for Inclusion. Developing learning and participation in schools. Bristol: CSIE.

Bretones, M., Flórez, M. A. y Rodríguez-Martín, A. (2018). Inclusión y universidad. Guia de orientaciones, adaptaciones y recursos para la comunidad universitaria. Recuperado de: https://www.unioviedo.es/ONEO/wp-content/uploads/2017/09/Inclusión-yUniversidad.-Gu\%C3\%ADa-de-orientaciones....pdf

Calderón, I. (2018). Deprived of human rights. Disability and Society, 33(10), 1666-1671. doi: $10.1080 / 09687599.2018 .1529260$

Castro, R. (2017). Revisión y análisis documental para estados del arte: imaginarios sociales sobre inclusión educativa. Revista Latinoamericana de Educación Inclusiva, 11(1), 183-297.

Ceballos, N. (2017). Guia rápida para promover la participación en las escuelas. Recuperado de: https://repositorio.unican.es/xmlui/handle/10902/13273

Ceballos, N. y Saiz, A. (2019). Promoviendo la participación del alumnado en la escuela. Análisis de materiales y guías internacionales para docentes que desarrollan experiencias de voz del alumnado. Profesorado. Revista de Currículum y Formación del Profesorado, 23(1), 330-350. doi: 10.30827/profesorado.v23i1.9157

Cobeñas, P., Fernández, C., Galeazzi, M., Noziglia, J., Santuccione, G. y Schnek, A. (2017). Educación inclusiva y de calidad, un derecho de todos. Recuperado de: https://grupoart24.org/downloads/publicaciones/manual_educacion_inclusiva.pdf

Comisión de Familias en Inclusión de Feaps. (2015). Guía informativa para familiares de alumnos y alumnas con necesidades educativas especiales. Madrid: Plena inclusión España.

Echeita, G. (2013). Inclusión y exclusión educativa. De nuevo, “voz y quebranto”. REICE. Revista Iberoamericana sobre Calidad, Eficacia y Cambio en Educación, 11(2), 99-118. 
Echeita, G. (2017). Educación inclusiva. Sonrisas y lágrimas. Aula Abierta, 46, 17-24. doi: 10.17811/rifie.46.2017.17-24

Echeita, G., Fernández-Blázquez, M. L. y Simón, C. (2019). Termómetro para la valoración de la educación inclusiva en un centro escolar. Madrid: Plena inclusión España.

Etxabe, E., López-Vélez, A. L. y Soria, L. (2018). Por una escuela que responda a las particularidades del alumnado y reconozca la capacidad de cada persona para aprender. Bilbao: FEVAS Plena Inclusión Euskadi.

Forteza, D., Fuster, L. y Moreno-Tallón, F. (2019). Barreras para el aprendizaje y la participación en la escuela del alumnado con dislexia: voces de familias. Revista Internacional de Educación para la Justicia Social, 8(2), 113-130. doi: 10.15366/riejs2019.8.2.006.

Fundación Plataforma Educativa. (2019a). Implementando la inclusión en las escuelas. Guía sobre cómo empezar a dirigir e implementar vuestro desarrollo escolar hacia la inclusión. Recuperado de: https://cdn.website-editor.net/7719483231fe4883a9297404e957bcce/ files/uploaded/Guia\%2520IIIS\%2520Espa\%25C3\%25B1ol.pdf

Fundación Plataforma Educativa. (2019b). Implementando la inclusión en las escuelas. Módulos de Capacitación para apoyar el desarrollo escolar inclusivo. Recuperado de: https://cdn.website-editor.net/7719483231fe4883a9297404e957bcce/files/uploaded/ Training\%2520Mod\%2520espv5.pdf

García, I., Romero, S., Sanz, M. P. y Martínez, P. (2012). Guía Práctica para la realización de Trabajos Fin de Grado y Trabajos Fin de Máster. EDITUM. Servicio de Publicaciones de la Universidad de Murcia.

García-SAnz, M. P. (2018). Algunas propiedades psicométricas de las guías para evaluar prácticas inclusivas en el aula. Revista Española de Orientación y Psicopedagogía, 29(2), 8-28. doi: 10.5944/reop.vol.29.num.2.2018.23150

González-Gil, F., Martín-Pastor, E. y Poy, R. (2019). Educación inclusiva: barreras y facilitadores para su desarrollo. Análisis de la percepción del profesorado. Profesorado. Revista de Currículum y Formación del Profesorado, 23(1), 243-263. doi: 10.30827/profesorado.v23i1.9153

Guirao, J. M. y Arnaiz, P. (2014). Instrumentos para la autoevaluación y la mejora de la atención a la diversidad en centros educativos. Siglo Cero, 45(4), 22-47.

Harris, A. y Jones, M. (2020). System Recall. Leading for equity and excellence in education. Thousand Oaks, CA: Corwin.

Knowledge and Human Development Authority. (2019). Implementing inclusive education: a guide for schools. Recuperado de: https://www.khda.gov.ae/Areas/Administration/ Content/FileUploads/Publication/Documents/English/20190123084554_SENDPOLICYSCHOOLS_EN.pdf

López, F. (2002). El análisis de contenido como método de investigación. XXI, Revista de Educación, 4, 167-179.

Medina, M. y Alcaín, E. (2018). Guía metodológica para el tránsito educativo de estudiantes con discapacidad a la universidad. Recuperado de: https://blogs.comillas.edu/fei/wpcontent/uploads/sites/7/2018/11/guia_metodologica_para_el_transito_educativo_de_estudiantes_con_discapacidad_a_la_universidad_1.pdf

Plena Inclusión. (2018). El camino hacia la Educación Inclusiva. Un análisis desde la perspectiva de los profesionales de la educación. Madrid: Plena inclusión.

Porter, G. y Towell, D. (2019). La ruta hacia la escuela inclusiva. Avanzando con la transformación escolar desde adentro. Recuperado de: https://www.centreforwelfarereform.org/ uploads/attachment/662/la-ruta-hacia-la-escuela-inclusiva.pdf

RoJAs, I. R. (2011). Elementos para el diseño de técnicas de investigación: una propuesta de definiciones y procedimientos en la investigación científica. Tiempo de Educar, 12(24), 227-297. 


\section{REVISIÓN DE GUÍAS PARA AVANZAR HACIA UNA EDUCACIÓN MÁS INCLUSIVA EN LOS CENTROS EDUCATIVOS \\ CECILIA AZORÍN ABELLÁN Y MÓNICA DEL MAR GONZÁLEZ BOTÍA}

Unesco. (2015). Educación 2030 Declaración de Incheon y Marco de Acción: hacia una educación inclusiva y equitativa de calidad y un aprendizaje a lo largo de la vida para todos. París: UNESCO.

Unesco. (2020). Global Education Monitoring Report. Inclusion and education: All means all. Paris: UNESCO.

Yarbrough, D., Brandt, J. y Moore, M. (2016). A Guide to Planning an Inclusion Project. Recuperado de: http://www.doe.virginia.gov/special_ed/transition_svcs/inclusionproject.pdf 


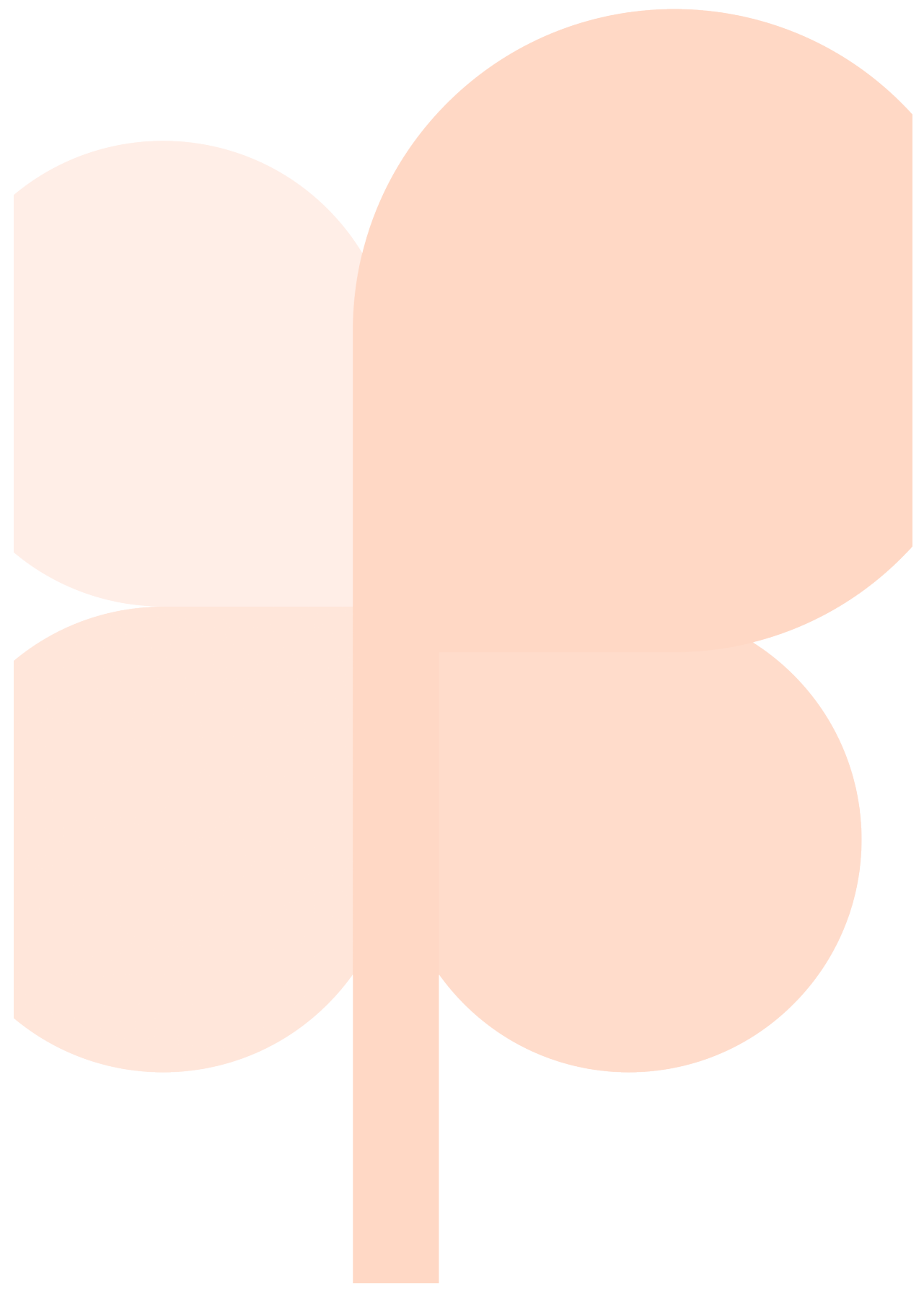

